\author{
Diana CelayaTentori* \\ Alejandro Díaz Bautista**
}

\title{
Crecimiento, Instituciones y Convergencia en MéXico CONSIDERANDO A LA FRONTERA NORTE
}

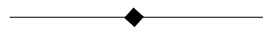

\section{RESUMEN}

El presente estudio se centra en uno de los problemas económicos regionales fundamentales de nuestra época: el desenvolvimiento económico de la convergencia y el crecimiento en las regiones considerando a las instituciones.

El estudio se ocupa de revisar la literatura teórica del crecimiento económico, y en particular por algunos de los desarrollos teóricos y empíricos que han tenido lugar recientemente en el campo de la convergencia económica.

De igual forma, el estudio analiza la convergencia regional en México y aborda los fundamentos teóricos y empíricos en el cual las instituciones juegan un papel importante en el proceso de integración regional en México y en especial en la frontera norte.

Finalmente, se realiza un análisis empírico de la convergencia regional de largo plazo para los estados de México, introduciendo una variable de corrupción dentro de las instituciones, una variable de frontera, controlando con una variable de capital humano regional. Los resultados estiman una tasa anual de convergencia de entre $-0.018 \%$ a $4.1 \%$ para el periodo de 1970 a 2001 , cuando se toman en cuenta los efectos de las variables institucionales, de capital humano y de frontera.

\section{ABSTRACT}

The present study is focused on one of the main economic problems of our time, the study of regional economic convergence and economic growth with consideration to institutions. The study conducts a literature review on the theoretical and empirical works of economic growth, with emphasis in regional economic convergence and institutions. The empirical and theoretical analysis is centered on the regional convergence of the Mexican states taking into consideration institutional and border state factors. The empirical analysis estimates an annual rate of convergence that varies from $-0.018 \%$ to $4.1 \%$ for the period between 1970 and 2001, when controlling for human capital, institutional and border effects.

* Catedrática en la Facultad de Economía de la Universidad Autónoma de Baja California.

** Profesor-Investigador de Economía, Departamento de Estudios Económicos,

El Colegio de la Frontera Norte: adiazbau@hotmail.com adiazbau@yahoo.com 


\section{INTRODUCCIÓN AL CRECIMIENTO ECONÓMICO REGIONAL}

El estudio del crecimiento económico regional es relativamente nuevo dentro de la economía mexicana. Algunas de las preguntas claves dentro de la economía regional mexicana pueden ser las siguientes. ¿Cuáles son los factores económicos e institucionales responsables de la convergencia o divergencia en las tasas de crecimiento económico en las regiones de México? ¿Por qué al gunas regiones de México, como las de la frontera norte, amplían sus economías y mejoran sus estándares de vida, mientras que otras han caído? ¿Cuál es el papel de las instituciones dentro del crecimiento económico regional? El propósito del estudio es contestar éstas y otras preguntas del campo de la economía regional mexicana.

La economía regional es una nueva área en la cual los economistas mexicanos pueden especializarse. La teoría del crecimiento regional sebasa en explicar, medianteun conjunto reducido defactores, los procesos de diferenciación regional. ${ }^{1}$ Las escuelas de pensamiento tradicionales como la clásica, neoclásica y keynesiana, conceden especial importancia a la movilidad de factores (capital y trabajo), y han sido la piedra angular mediante la cual se ha generado el desarrollo teórico y el diseño de políticas regionales en las últimas décadas en todo el mundo.

Los pensadores clásicos de la ciencia económica como Adam Smith y David Ricardo, reconocieron el papel de la tecnología y las instituciones en el crecimiento, pero pensaban que la agricultura, el crecimiento de la fuerza laboral y la formación de capital eran los factores más importantes en la función de producción.

El teorema de la convergencia de los factores de precio y el teorema de igualación precio-factor, han sido herramientas para los teóricos regionales comerciales desde que Eli Heckscher y Bertil Ohlin realizaron sus contribuciones seminales en 1919 y 1924 . El paradigma de Heckscher-Ohlin discute que los países exportan las mate-

${ }^{1}$ ¿Por qué algunas regiones se vuelven ricas y otras pobres? Ésta es una de las preguntas fundamentales de la economía mundial. 
rias donde utilizan los factores intensivos y bien dotados en su región, mientras que importan las materias intensivas en factores en donde se encuentran mal dotados. Esta forma de producción y exportación puede fomentar el crecimiento de un país.

El crecimiento de los factores primarios de producción como el capital y el trabajo son un componente dominante en el crecimiento del producto regional, y es esencial para explicar los modelos del crecimiento de los economistas clásicos. La mayoría de los economistas de la primera mitad del siglo XX tratan a las instituciones como un factor exógeno sin explicarlo.

La teoría general de J. Maynard Keynes, con toda su insistencia respecto a la relación del crecimiento con el dinero, las finanzas y las expectativas, sirve con eficacia para explicar los eventos antes de 1936. Esta misma teoría, sin embargo, aunque considera al mercado como creador del crecimiento y de los ciclos, no considera a las instituciones como un factor importante en el crecimiento económico regional (cuadro 1 ).

La economía keynesiana del crecimiento fue desarrollada por Roy F. Harrod y J. Hicks (quienes estaban en Oxford), con la adopción subsecuente del programa de investigación por los keynesianos de la Universidad de Cambridge (Nicolás Kaldor, Joan Robinson y Richard Goodwin). Estas dos corrientes conforman la escuela de pensamiento conocida como Oxbridge, la cual dio lugar a la revolución keynesiana enfocada al crecimiento económico.

Hay tres partes importantes del programa de investigación de Oxbridge: 1) el desarrollo de las teorías del acelerador en el multiplicador keynesiano del ciclo considerando al ahorro; 2) el desarrollo de ciclos endógenos vía mecanismos no lineares; y 3) el desarrollo del crecimiento de tipo keynesiano. El lazo del acelerador en el multiplicador fue introducido por Roy F. Harrod en su teoría de ciclos, y después readaptado por Harrod en la teoría del crecimiento. Sin embargo, aunque estos modelos amplían las estructuras no lineares de los modelos lineares del multiplicador-acelerador, carecen de la referencia de las instituciones como determinante del crecimiento. 
CUADRO 1 Resumendelosmodelosdecredimientoeconómico-regional.

\begin{tabular}{|c|c|c|c|}
\hline & $\begin{array}{c}\text { Teorías, modelos } \\
\text { y principales } \\
\text { exponentes } \\
\end{array}$ & Hipótesis & Supuestos \\
\hline Clásico & $\begin{array}{l}\text { Smith, Ricardo, } \\
\text { Marx, Hecks- } \\
\text { cher-Ohlin }\end{array}$ & $\begin{array}{l}\text { División del } \\
\text { trabajo }\end{array}$ & $\begin{array}{l}\text { El crecimiento depende de la } \\
\text { tasa de crecimiento de la po- } \\
\text { blación }\end{array}$ \\
\hline siano & $\begin{array}{l}\text { Keynesianos y } \\
\text { Postkeynesianos } \\
\text { (Keynes, Harrod, } \\
\text { Domar, Kaldor, } \\
\text { Robinson, neo- } \\
\text { keynesianos) }\end{array}$ & $\begin{array}{l}\text { (Ciclos largos, } \\
\text { multiplicador } \\
\text { y acelerador } \\
\text { con dependen- } \\
\text { cia del camino) }\end{array}$ & $\begin{array}{l}\text { El crecimiento depende de la } \\
\text { tasa de ahorro. Concurren- } \\
\text { cia imperfecta y rendimien- } \\
\text { tos crecientes }\end{array}$ \\
\hline $\mathrm{Neo}$ & \begin{tabular}{|l} 
Neoclásicas de \\
crecimiento y \\
movilidad de \\
factores (Solow, \\
Ram-sey, Swan)
\end{tabular} & $\begin{array}{l}\text { La reestructu- } \\
\text { ración de los } \\
\text { sistemas de la } \\
\text { producción y } \\
\text { del trabajo }\end{array}$ & $\begin{array}{l}\text { El crecimiento en el largo pla- } \\
\text { zo depende del progreso tec- } \\
\text { nológico, rendimientos cons- } \\
\text { tantes y rendimiento decre- } \\
\text { ciente del capital, así como } \\
\text { de la tasa de incremento } \\
\text { poblacional y depreciación } \\
\text { del capital }\end{array}$ \\
\hline $\begin{array}{l}\text { Nueva } \\
\text { economía } \\
\text { geográfica }\end{array}$ & $\begin{array}{l}\text { ( K r u g m a } \mathrm{n}, \\
\text { Fujita, Venables } \\
\text { y Porter) }\end{array}$ & $\begin{array}{l}\text { Producción } \\
\text { flexible, siste- } \\
\text { mas complejos } \\
\text { y nuevo espa- } \\
\text { cio industrial }\end{array}$ & $\begin{array}{l}\text { Competencia monopolística, } \\
\text { costos de transporte, rivali- } \\
\text { dad, condiciones de los fac- } \\
\text { tores, condiciones de deman- } \\
\text { da, clusters industriales }\end{array}$ \\
\hline Endógeno & $\begin{array}{l}\text { Nuevas teorías } \\
\text { neoclásicas del } \\
\text { crecimiento o } \\
\text { crecimiento en- } \\
\text { dógeno (Man- } \\
\text { kiw, Romer, Ba- } \\
\text { rro, Lucas) }\end{array}$ & $\begin{array}{l}\text { Externalidades } \\
\text { en los sistemas } \\
\text { de la produc- } \\
\text { ción, institucio- } \\
\text { nes, conoci- } \\
\text { miento y el tra- } \\
\text { bajo }\end{array}$ & $\begin{array}{l}\text { El crecimiento de largo pla- } \\
\text { zo depende de la acumula- } \\
\text { ción de capital físico, de ca- } \\
\text { pital humano, de las insti- } \\
\text { tuciones y de conocimientos. } \\
\text { Explicadas endógenamente } \\
\text { en función de expectativas } \\
\text { de ganancias, externalidades } \\
\text { y rendimientos crecientes }\end{array}$ \\
\hline
\end{tabular}


A finales de la década de los cincuenta surgieron las teorías neoclásicas del crecimiento. Para entenderlas es necesario comenzar con el modelo simple de crecimiento. Considere la producción de una empresa $(Y)$, en función de tres variables: el capital $(K)$, el trabajo $(L)$ y el conocimiento o la eficacia del trabajo,(A ); así podemos observar la siguiente función:

$$
Y=K^{a}\left(A_{t} L\right)^{1-a} \quad 0<a<1
$$

Los exponentes a y (1-a) miden la contribución relativa de los dos factores en las entradas de información, capitales y eficacia de la función de producción. El sumar a la unidad los exponentes es muestra del supuesto de retornos a escala constantes en la producción (por ejemplo, si todas las entradas de factores se doblan simultáneamente éste conducirá a doblar exactamente la producción).

La teoría neoclásica del crecimiento en la tradición de Solow (1956), está basada en los supuestos de mercados competitivos, donde todos los factores de la producción serían recompensados según su contribución marginal social a la producción. El modelo de Solow (1956) exhibe retornos constantes a escala en la producción y una productividad marginal decreciente en los dos factores de la producción, los cuales incluyen el trabajo homogéneo y del capital físico. El aumento en la relación capital-trabajo incrementa la cantidad de capital por trabajador, la productividad y el ingreso per cápita. El índice de la inversión y las tasas de crecimiento de la mano de obra, exógena al modelo, son determinados por el ahorro y la tasa de crecimiento de la población, respectivamente. El aumentar el índice de la inversión más allá del índice del crecimiento de la población (y por lo tanto, mayor mano de obra), ocasiona que la relación capitaltrabajo de transformación nos lleve a un mayor nivel de crecimiento económico.

El modelo neoclásico del crecimiento predice que convergerán las tasas de crecimiento de varias regiones en el largo plazo. En un ambiente de mercado libre, cada país tendrá acceso a las tecnologías similares y los factores altamente móviles en la producción 
serán utilizados en las regiones que son capaces de generar más rentas. Las regiones más pobres (dadas su posición inicial) están en condiciones mejores para explotar los aumentos de capital, puesto que tienen un nivel relativamente bajo de capital trabajo en la relación de transformación. Mientras que las regiones con al tos índices de crecimiento de la población deben presentar un crecimiento per cápita más lento del producto regional. De igual forma, el aumento del índice de la inversión aumentará el capital y, por lo tanto, obtendrá mayores tasas de crecimiento.

Las críticas principales a la teoría neoclásica son a causa del especial hincapié en los conceptos de economías a escala y externas, en la ausencia de cambio tecnológico, en los rendimientos decrecientes del capital, y en las economías externas. Todos sus críticos se apoyaron en una contrapropuesta basada en un enfoque de demanda tipo keynesino. Estos críticos desarrollaron model os como el decrecimiento acumulativo (Hirshman, 1958), y de polos de crecimiento y ciclos de desarrollo (Hansen, 1950). Estos modelos suponen que en las regiones existen diferentes dotaciones de recursos productivos y de otros factores económicos y extraeconómicos, y sostienen que las relaciones interregionales son las responsables de las disparidades económicas y tratan de explicar que éstas se agravan en vez de corregirse. En el cuadro 1 se muestran las principales teorías y exponentes del crecimiento económico regional.

Una de las áreas más interesantes de la economía regional contemporánea, que surge después de la teoría neoclásica, es la nueva geografía económica. Esta literatura económica fue retomada por Krugman (1995), y se refiere a la ubicación de la actividad económica regional considerando un modelo de competencia monopolística para explicar las disparidades regionales del crecimiento.

Krugman menciona que la aparición de la actividad económica no es al azar, pero si es caótica. Este autor utiliza modelos basados en los principios del caos y de la dinámica no lineal. En su modelo de alta tecnología incluye variables como los sistemas del transporte y de comunicaciones y sus costos.

La literatura de la geografía económica se ha centrado en los costos de transporte, el conocimiento tecnológico y su crecimiento, 
una vez que haya ocurrido una aglomeración en la actividad económica. Por otra parte, pero en el mismo sentido, Porter (1990) nos muestra las ventajas de la ubicación de la actividad económica re gional en clusters industriales, siendo las aglomeraciones regionales las mayores fuentes del crecimiento de las naciones.

La nueva geografía económica ofrece la promesa de combinar las teorías económicas regionales con una fundación teórica más rigurosa. Sin embargo, el papel de las instituciones queda fuera de los modelos.

Krugman, Fujita y Venables (1999) vuelven a los modelos de la natural eza tipo evolutivos para ver quéestrategias emplean los agentes para transformar y fomentar el crecimiento regional. Este tipo de modelos estudia a la economía como sistema complejo de desarrollo basado en supuestos de redes no lineales adaptantes que caracteriza las redes en los procesos económicos, y analiza las estructuras que emergen al aplicarse a la economía. El juego científico económico se centra en modelar a los autómatas celulares para entender cómo las reglas simples que gobiernan el comportamiento de células individuales pueden afectar el comportamiento de sus vecinos más cercanos produciendo nuevos modelos de aglomeraciones para la economía regional.

Por otro lado, se presenta otra línea de investigación donde cada vez más se observa la débil movilidad de los factores capital y trabajo, y la nueva noción de las instituciones en el proceso de transformación social, dando paso a una nueva aproximación al desarrollo regional denominado enfoque endógeno. Consecuentemente, el modelo de crecimiento endógeno se centra en el análisis de los factores institucionales regionales, mientras que los modelos de crecimiento neoclásico y keynesiano atribuían un papel secundario a los factores institucionales, considerando como relevantes únicamente los costos laborales y las economías a escala. Explícitamente se endogeniza el papel de las instituciones y del cambio técnico en el modelo. Recordemos que la teoría tradicional del crecimiento trató este fenómeno como exógeno al modelo.

Finalmente, al igual que el modelo neoclásico, el modelo de crecimiento endógeno resal ta el lado dela oferta de la economía regional. 
Por su interés en la evolución cualitativa de los recursos locales, tanto humanos como empresariales y de capital, y en la efectividad local de las instituciones a nivel local, el modelo de crecimiento endógeno parece apuntar hacia una intervención activa. En este aspecto es similar al modelo keynesiano, que tenía más confianza en el papel del gobierno y dela inversión autónoma empresarial que en los mecanismos automáticos del mercado. El modelo de crecimiento endógeno atribuye más importancia a las instituciones locales que a las políticas regionales implementandas desde el centro. Sus seguidores promueven una autonomía administrativa más generosa de las regiones, al igual que programas de desarrollo regional y estatal para todas las regiones.

\section{EL CRECIMIENTO ECONÓMICO Y LA CONVERGENCIA}

La literatura reciente sobre crecimiento económico y convergencia ha acuñado dos términos que han acabado por popularizarse dentro del campo de la economía regional mexicana. Estos dos términos son la convergencia beta y la convergencia sigma, y cada uno de ellos corresponde con una definición de convergencia real. Así, por ejemplo, al considerar la variable del PIB real per cápita para un conjunto dado de economías (países, regiones, estados, etcétera), y para un periodo determinado (normalmente un número de años lo suficientemente largo como para poder hablar de crecimiento a largo plazo), se dice que existe convergencia si se obtiene una relación inversa entre el crecimiento medio anual del PIB per cápita y el nivel de PIB per cápita del año inicial.

De los estudios empíricos realizados en los últimos años sobre el área de la convergencia, se observa un hecho estilizado del crecimiento, donde los grupos de economías consideradas como homogéneas o similares, en virtud de algún tipo de criterio económico/ institucional, presentan el fenómeno de convergencia real a largo plazo.

Lo anterior es una manifestación del Ilamado fenómeno de convergencia relativa o condicional, donde existe convergencia sólo 
entre conjuntos de economías que comparten una serie de variables o entre economías que pertenecen a un club de la convergencia.

En este caso se dispone de dos líneas teóricas bien definidas, que son las relacionadas con el modelo de crecimiento neoclásico de Solow (1956) y con el modelo de catch-up tecnológico de A bramovitz (1986), que conducen a la misma predicción. Se puede observar cierta convergencia, pero tan sólo entre grupos de economías con una serie de rasgos en común (convergencia condicional). Si suponemos dos países con características económico-institucionales similares y un nivel tecnológico mundial dado, en el caso del modelo neoclásico, lo que sucede es que el país más atrasado (que se caracteriza por tener un menor stock de capital per cápita) crece más queel más avanzado porque, por la ley de los rendimientos decrecientes, la productividad marginal de su stock de capital será mayor que la del país avanzado (es decir, $(\mathrm{K} / \mathrm{L})_{\mathrm{P}}<(\mathrm{K} / \mathrm{L})_{R}, \mathrm{PM} \mathrm{gK}_{\mathrm{P}}>\mathrm{PMgK} \mathrm{K}_{\mathrm{R}}$ ), debido a que en el país atrasado es mayor la rentabilidad del capital.

En el modelo de catch-up tecnológico uno de los supuestos explícitos es que las dos economías tienen niveles institucionales y tecnológicos diferentes (funciones de producción diferentes), de modo que la difusión internacional de la tecnología debido a las instituciones, desde el país líder hacia el país seguidor, es lo que explica que se dé convergencia. En definitiva, en el primer caso el fenómeno de la convergencia se explica a partir de la ley de los rendimientos decrecientes aplicados al capital, en tanto que en el segundo se produce por el efecto de la difusión internacional de los conocimientos, tratándose en los dos casos de un fenómeno de convergencia condicional.

La literatura sobre la relación entre instituciones, política económica y crecimiento a largo plazo ha despertado un notable interés en los últimos años. Sin embargo, si algo destaca en este tipo de estudios es la gran dispersión de enfoques y líneas teóricas existentes. North (1993) ha definido a las instituciones como cualquier restricción socialmente impuesta sobre el comportamiento humano. La definición, dentro de la economía regional mexicana, de la naturaleza y efectos de las instituciones en el crecimiento y convergencia regional, es extremadamente importante. En México las 
restricciones socialmente impuestas y las instituciones limitan la actividad económica. Un desarrollo exitoso de políticas públicas en México necesita de un mejor entendimiento de las dinámicas del cambio económico considerando a las instituciones, si es que se desea que dichas políticas tengan los resultados necesarios. Aun cuando todavía existe un largo camino que recorrer para obtener dicho modelo, la estructura que está desarrollándose en la nueva economía institucional en México sugiere políticas de crecimiento radicalmente diferentes de aquellas propuestas por economistas tradicionales de desarrollo mexicanos.

En el enfoque de Olson (1996) se denominan instituciones estables a aquéllas que garantizan los derechos individuales a la propiedad y el cumplimiento de los contratos a través de las generaciones. Un ejemplo serían los países industrializados con economías desarrolladas, las cuales pueden ser el prototipo de régimen institucional que permite alcanzar un máximo desarrollo a largo plazo.

Esta hipótesis ha sido sometida a contraste desde las primeras regresiones de convergencia efectuadas por Barro y Sala-i-Martin (1991). Así, por ejemplo, Barro (1991), para una muestra mundial de países, utilizó como variables proxy de la inestabilidad políticoinstitucional el número de revoluciones y golpes de Estado durante el periodo analizado (1960-1985), y el número de asesinatos por cada mil habitantes y año, obteniendo una relación negativa entre ellas y el crecimiento per cápita para el periodo mencionado. Para México, los estudios de convergencia económica considerando a las instituciones no han sido considerados.

\section{EVIDENCIA EMPÍRICA DE LA CONVERGENCIA \\ Y LAS INSTITUCIONES MEXICANAS}

En el transcurso de la última década, el campo más fértil dela investigación macroeconómica en México y en todo el mundo ha sido, sin lugar a dudas, la teoría del crecimiento económico, y al interior de éste, la polémica más intensa se ha dado en torno a la existencia (o no) de convergencia entre diferentes regiones. 
Los defensores del modelo neoclásico predicen convergencia, mientras que los defensores de los nuevos modelos de crecimiento endógeno indican que ésta no se puede dar, dado que los factores no presentan rendimientos decrecientes. La discusión fue rápidamente llevada al terreno empírico, y a partir fundamentalmente de los trabajos de Barro y Sala-i-Martin (1992) y Mankiw, Romer y Weil (1992), se han elaborado cientos de artículos al respecto, encontrando en su mayoría que si bien no se puede hablar de convergencia absoluta entre países, sí existe convergencia condicional entre ellos.

Los diversos acercamientos teóricos discutidos han motivado al autor a pensar en la posibilidad de un número de efectos de las externalidades que se presentan en las instituciones, educación general y en otras variables como el índice de desarrollo humano de las regiones. Para identificar externalidades institucionales intertemporales, quisiéramos idealmente observar diversas regiones $\mathrm{y} / \mathrm{o}$ el tiempo-periodo en que estas externalidades estuvieran presentes para poder comparar los funcionamientos del crecimiento. Identificar la existencia y el fragmento de las externalidades en las instituciones (mediante la corrupción de las mismas), la educación y otras variables en la evidencia macrorregional, presenta dificultades en la práctica, y hasta las metodologías y los datos utilizados en los pocos estudios empíricos de este tipo son difíciles de obtener. La mayoría de la evidencia entre países viene del análisis de corte transversal con muestras de los países de la OCDE en el periodo posterior a 1960.

La convergencia per cápita del PIB entre las regiones, según lo predicho por la teoría tradicional del crecimiento, no ha podido materializar una respuesta verdaderamente única en los ámbitos global y regional. Si hubiera ocurrido la convergencia, esperaríamos ver una tendencia a la baja de los ingresos iniciales más altos comparados con las tasas de crecimiento de las regiones pobres, o veríamos que los países se acercan en términos de ingreso per cápita y nivel de vida.

A partir del periodo 1950-1980, la convergencia tiende a ocurrir, pero no entre todos los países. Las naciones industrializadas, las economías central-planeadas y los países en vías de desarrollo 
experimentan una convergencia de tipo intragrupo, formando bloques de regiones de convergencia, o lo que en la literatura económica contemporánea se denomina clubes de convergencia.

Mankiw, Romer y Weil (1992), en su estudio sobre la contribución de la educación al crecimiento económico, mencionan que el modelo neoclásico de crecimiento económico de Solow -incluyendo al capital humano- provee una guía satisfactoria del proceso de conocimiento en el crecimiento económico entre las naciones del mundo. Se reporta que $80 \%$ de las variaciones internacionales del ingreso per cápita en el ingreso se puede explicar por el modelo de Solow, aumentado con la educación.

Mankiw (1995) provee los argumentos sobre la evidencia empírica y su consistencia con las predicciones del modelo en términos de los efectos en la inversión, capital físico y humano, y el crecimiento de la población en el nivel de producción. También menciona quesi el modelo está bien especificado, se predice la convergencia condicional. Este fenómeno de la convergencia tiene una amplia aceptación en estudios de crecimiento muy bien documentados como los de De Long (1988), Dowrick y N guyen (1988) y Barro y Sala-i-Martin (1991).

Para México se ha comprobado empíricamente la convergencia entre sus entidades federativas. Asimismo, la mayoría de los estudios existentes sobre el tema han encontrado evidencia de al gún tipo de convergencia entre las regiones de México. Algunos trabajos sobre convergencia en nuestro país realizados en los últimos años son los de Esquivel (1999), Díaz Bautista (2000) y Messmacher (2000).

Los resultados empíricos para México muestran cierto grado de convergencia. Para la relación entre la tasa de crecimiento del PIB per cápita y el nivel original del PIB, Esquivel (1999) muestra evidencia de un proceso de convergencia de 1.6\% entre 1940 y 1995, mientras que Messmacher (2000) menciona que existe cierta evidencia empírica a favor de un proceso de convergencia de 1970 a 1980, mismo que se acelera en el periodo 1980-1985; sin embargo, el proceso se revierte durante el periodo 1985-1993, y se vuelve a observar una débil evidencia de convergencia durante la década de los noventa. 
La regresión de convergencia para el periodo 1970-1999, proporciona escasa evidencia de que se hayan cerrado las disparidades regionales de ingreso durante todo este periodo. Al controlar con las diferencias interregionales en capital humano, Díaz Bautista (2000) obtiene evidencia empírica que muestra un proceso de convergencia entre los estados de México para el periodo 1970-1995. Como se muestra desde el punto de vista metodológico, los modelos neoclásicos y endógenos son modelos de teoría positiva de crecimiento en el sentido de que explican y utilizan variables que se pueden observar y medir. Los estudios anteriores de convergencia en México han dejado fuera el papel de las instituciones en el crecimiento regional. Para el presente estudio se utilizan las bases de los modelos neoclásicos y endógenos, y se incluyen variables que reflejan la efectividad de las instituciones para fomentar el crecimiento económico de un país (cuadro 2).

Aunque las teorías económicas del crecimiento predicen la convergencia, la evidencia empírica está sujeta al debate de la convergencia contra la divergencia. De acuerdo con la metodología de Mankiw (1993) y Barro y Sala-i-Martin (1995), y siguiendo el estudio de Díaz Bautista (2001), el modelo neoclásico estándar de crecimiento se deriva de la función de producción con retornos constantes a escala considerando dos insumos: el capital y el trabajo. La producción ${ }^{2}$ en cualquier punto está dada por:

$$
Y(t)=K(t)^{\alpha} H(t)^{\beta}(A(t) L(t))^{1-\alpha-\beta}
$$

\footnotetext{
${ }^{2}$ Donde $Y, K, H$ y L son producción, capital físico, capital humano y trabajadores, respectivamente, a es la elasticidad parcial de la producción con respecto al capital físico, $\beta$ es la elasticidad parcial de la producción con respecto al capital humano, y A (t) es el nivel de eficiencia económico y tecnológico. Se puede asumir que este nivel de eficiencia $A(t)$ tiene dos componentes: la eficiencia económica I (t) la cual depende de las institucioness y políticas económicas, y el nivel de progreso tecnológico $\Omega$ (t), como en Cellini et al. (1997). Así, I (t) puede ser escrito como una función logarítmica lineal de las variables institucionales y de políticas económicas, mientras que $\Omega(\mathrm{t})$ se asume crece a una tasa $\mathrm{g}(\mathrm{t})$.
} 
Las variables del lado derecho de la ecuación en el tiempo (de aquí en adelante las variables con puntos serán representativas de esta descripción) ${ }^{3}$ se describen mediante:

$$
\begin{aligned}
& k^{\&}(t)=s_{k}(t) A(t)^{1-\alpha-\beta} k(t)^{\alpha} h(t)^{\beta}-(n(t)+d) k(t) \\
& \&(t)=s_{h}(t) A(t)^{1-\alpha-\beta} k(t)^{\alpha} h(t)^{\beta}-(n(t)+d) h(t) \\
& A(t)=I(t) \Omega(t) \\
& \ln I(t)=p_{0}+\sum_{j} p_{j} \ln V_{j}(t) \\
& S \&(t)=g(t) \Omega(t) \\
& \&(t)=n(t) L(t)
\end{aligned}
$$

Bajo el supuesto de retornos decrecientes en factores reproducibles, el sistema de ecuaciones se resuel ve para obtener los val ores de estado estable de $k^{*}$ y $h^{*}$ definidos por:

$$
\begin{aligned}
& \ln k^{*}(t)=\ln A(t)+\frac{1-\beta}{1-\alpha-\beta} \ln s_{k}(t)+\frac{\beta}{1-\alpha-\beta} \ln s_{h}(t)-\frac{1}{1-\alpha-\beta} \ln (g(t)+n(t)+d) \\
& \ln h^{*}(t)=\ln A(t)+\frac{\alpha}{1-\alpha-\beta} \ln s_{k}(t)+\frac{1-\alpha}{1-\alpha-\beta} \ln s_{h}(t)-\frac{1}{1-\alpha-\beta} \ln (g(t)+n(t)+d)
\end{aligned}
$$

Al sustituir estas dos ecuaciones en la función de producción y tomando sus logaritmos, obtenemos la expresión para el estado estable de la producción en su forma intensiva. ${ }^{4}$ Aquí utilizamos el promedio de años de escolaridad de la población económicamente activa como proxy del capital humano. El estado estable de la producción en su forma intensiva puede ser descrito de la siguiente manera:

${ }^{3}$ Como en la literatura tradicional de crecimiento $k=K / L, h=H / L, y=Y / L$, que muestran la razón capital-trabajo, producción de capital humano y producción por trabajador promedio, respectivamente, $s_{k}$ and $s_{h}$ son la tasa de inversión en capital físico y humano respectivamente, y d es la tasa de depreciación. Suponemos retornos decrecientes en factores reproducible $(\alpha+\beta<1)$.

${ }^{4}$ Lo último puede ser expresado ya sea como función de $s_{h}$ (inversión en capital humano) y las otras variables, o como función de $h^{*}$ (el stock de capital humano del estado estable) y las otras variables. 
Sin embargo, el estado estable del stock de capital humano no es observado. Como se muestra, la expresión para h* como función del capital humano real ${ }^{5}$ es:

$$
\ln h^{*}(t)=\ln h(t)+\frac{1-\psi}{\psi} \Delta \ln (h(t) / A(t))
$$

La ecuación [4] podría ser una especificación válida en el análisis empírico entre estados sólo si éstos estuviesen en sus estados estables correspondientes o si las desviaciones del mismo son independientes e idénticamente distribuidas. Si las tasas de crecimiento incluyen dinámicas fuera del estado estable, entonces estas dinámicas transitivas deberán ser modeladas explícitamente. Una aproximación lineal de estas dinámicas, similar a la de Mankiw et al. (1992),

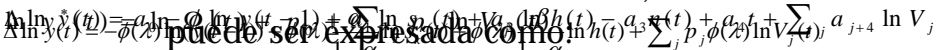

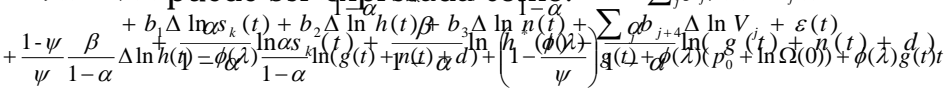

donde. $\lambda=(1-\alpha-\beta)(g(t)+n(t)+d)$

Al incluir las dinámicas de corto plazo a la ecuación [6] nos da: 
CUADRO2. Resultadosestimados delavelocidad deconvergenciaen el ingreso per cápitaentrelosestados deM éxico, condicional alasinstituciones 1970-2001, fronteranortey al capital humano.Variabledependiente:Tasadecreamiento del productoper cápita1970-2001.

\begin{tabular}{|l|c|c|c|c|}
\hline & $\mathrm{A}$ & $\mathrm{B}$ & $\mathrm{C}$ & $\mathrm{D}$ \\
\hline Constante & 0.6029 & 0.634 & 0.160 & 1.044 \\
& $(4.873)$ & $(4.235)$ & $(0.220)$ & $(3.614)$ \\
Log PIB 1970 & $-0.160)^{*}$ & $-0.149^{*}$ & $-0.414^{*}$ & $0.0184^{*}$ \\
Corrupción en & $(3.240)$ & $(2.543)$ & $(3.557)$ & $(5.06)$ \\
instituciones & -0.00706 & -0.007064 & $-0.010^{*}$ & $-0.011^{*}$ \\
Capital humano & $(1.385)$ & $(1.364)$ & $(2.795)$ & $(2.89)$ \\
a nivel primaria & & -1.00757 & -5.281 & -5.281 \\
Capital humano a & & $(-0.387)$ & $(-1.962)$ & $(-0.213)$ \\
nivel secundaria & & & -0.759 & -0.091 \\
Índice de desarrollo & & & $(-0.323)$ & $(-0.315)$ \\
humano & & & $3.298^{*}$ & $-2.18^{*}$ \\
Frontera norte & & & $(3.057)$ & $(1.71)$ \\
& & & & $-0.096^{*}$ \\
R Cuadrada ajustada & 0.219 & 0.196 & 0.175 & 0.482 \\
E.S. de la regresión & 0.106 & 0.107 & 0.109 & 0.072 \\
Log likelihood & 27.97 & 28.05 & 28.23 & 41.75 \\
F statistic & 5.36 & 3.52 & 3.65 & 6.78 \\
\hline
\end{tabular}

*Significancia a un nivel de $5 \%$.

(Estadísticos T en paréntesis).

La ecuación [7] representa la forma funcional genérica estimada empíricamente en el presente estudio, incluyendo la variable institucional. ${ }^{6}$ Las estimaciones de los coeficientes del estado estable, así como los parámetros de la función de producción, pueden ser obtenidos mediante los coeficientes estimados en esta ecuación al compararlos con la ecuación [6]. La ecuación [7] es importante debido a

${ }^{6}$ Donde $\Delta \ln y(t)$ es el cambio en el logaritmo del ingreso per cápita, In y(t-1) es el logaritmo de ingreso en el periodo $t-1, s_{k}(t)$ representa la tasa de inversión en capital, In h(t) es el logaritmo del capital humano promedio, $n(t)$ es el crecimiento de la población, t es un periodo, $\mathrm{Vj}$ es la variable institucional, los cambios en variables se reflejan en:

y $\varepsilon(t)$ es el término de error.

$$
\Delta \ln s_{k}(t), \Delta \ln h(t), \Delta \ln n(t), \sum \Delta \ln V_{j}
$$


que se puede analizar la relación entre el crecimiento económico y las instituciones mediante un proxy de corrupción en las instituciones y otras variables económicas siguiendo el estudio de Mauro (1995). Se efectúa un análisis econométrico al estilo de los modelos de convergencia, donde el cambio de crecimiento económico se encuentra condicionado sobre la corrupción, controlando con el nivel de capital humano, el índice de desarrollo humano y el crecimiento de la población, utilizando regresiones de mínimos cuadrados generalizados y variables instrumentales.

Las variables empíricas que se utilizan en el estudio se construyen de la siguiente manera: El crecimiento es el incremento porcentual anual del PIB per cápita ponderado sobre el periodo 1970-2001. La producción inicial es el PIB per cápita en 1970 y el periodo final del estudio para el año 2001 que proviene de las estimaciones del INEGI en sus reportes y en la página electrónica del instituto. La información sobre la producción y población se obtuvieron de las estadísticas del INEGI, mientras que la información sobre índices de desarrollo humano provienen del United Nations Development Programme, Human Development Report y del Banco Mundial en sus estudios W orld D evelopment Indicators y W orld D evelopment Report 1997: The State in a Changing W orld. El índice de desarrollo humano es una medida de calidad de vida que utiliza las Naciones Unidas y que está compuesto por indicadores de esperanza de vida, el nivel de capital humano de los adultos y el ingreso per cápita de las regiones. Para el capital humano se utiliza un proxy usando la proporción de la población que cursa la educación básica y secundaria, con estadísticas disponibles en las paginas electrónicas de la SEP y el INEGI.

La variable de corrupción viene de los estudios de W orld Bank. $\mathrm{H}$ elping Countries Combat Corruption y World Bank A nticorruption in Transition, y Transparencia M exicana. Indicadores de corrupción y buen gobierno. La variable de corrupción se refiere a la corrupción en las instituciones, y se calculó mediante un íncide de burocracia en los trámites realizados dentro de las instituciones a nivel nacional, por entidad federativa. El índice utiliza una escala que va de 0 a 100, donde entre menor sea el índice se tiene menor corrupción durante 
la transacción del trámite realizado. En el índice se muestra que la ciudad de México es la entidad con mayor corrupción, y Colima el estado con menor corrupción en términos institucionales de trámites burocráticos. La variable de frontera es una variable dummy para los estados que son considerados de la frontera norte de M éxico con Estados Unidos.

La ecuación de convergencia a estimar empíricamente será la siguiente:

$\Lambda \ln y(t)=\beta 0+\beta 1$ PIB per cápita inicial $+\beta 2$ capital humano $+\beta 3$ índice de desarrollo humano $+\beta 4$ variable institucional de la corrupción $+\beta 4$ variable institucional de frontera $+\varepsilon$

Se realizan cuatro estimaciones que contemplan las regresiones de convergencia controlando la variable de corrupción; la variable de corrupción y la de educación primaria; la variable de corrupción y la de educación primaria y secundaria; y final mente la variable de corrupción, las variables educativas y la variable del índice de desarrollo humano. Se muestra que el crecimiento del ingreso per cápita está relacionado con el nivel de educación, el desarrollo humano y las instituciones presentes en los diversos estados del país. Se puede pensar que al gunas variables tienen un efecto de doble causalidad entre ellas, por lo que el método econométrico es de mínimos cuadrados generalizados con instrumentos para el periodo 1970-2001. En el cuadro 2 se muestran los resultados empíricos de las regresiones de convergencia tomando en cuenta la corrupción dentro de las instituciones.

Para México se ha comprobado empíricamente la teoría del modelo neoclásico de crecimiento y se observa evidencia de convergencia entre los estados de nuestro país para el periodo 1970-2001, lo que significa que deberá esperarse una tendencia a cerrar la brecha entre los estados ricos como el Distrito Federal, Baja California, Jalisco y Nuevo León, y los estados pobres, como Chiapas, Oaxaca y Guerrero. El resultado de la regresión A por mínimos cuadrados generalizados para la república mexicana muestra una B con el signo negativo esperado y estadísticamente significativo (al 95\%), 
por lo que se confirma la existencia de convergencia regional considerando la variable de corrupción y las variables educativas. El tiempo de convergencia entre ellos, considerando las variables antes mencionadas, se estima sea aproximadamente igual a la vida media nacional (de 40 años). Los resultados muestran que tendría que pasar una generación completa para ver este resultado de convergencia a mediados del siglo XXI.

Para el periodo 1970-2001, el parámetro de convergencia condicional en la regresión C es significativo y negativo con $-0.414 \%$. Los resultados indican que las regiones de México Ilegarán a un determinado ingreso de largo plazo al considerar las variables de corrupción y de capital humano. En este periodo, México tuvo tasas de crecimiento de ingreso per cápita negativas, y un menor porcentaje de la población que fue a primaria y secundaria (de los 5 a los 14 años de edad). Al considerar el modelo tradicional de convergencia absoluta afirmamos la existencia de convergencia absoluta que marca una relación inversa entre el nivel inicial y la tasa de crecimiento del ingreso per cápita, e indica, por tanto, que los estados más pobres de la república tienden a alcanzar a los más ricos, tomando en cuenta el capital humano de cada estado y observando que los primeros están creciendo más que los segundos.

El resultado de la regresión por mínimos cuadrados generalizados para la república mexicana encontró un valor de convergencia con el signo esperado y estadísticamente significativo (al 95\%). En la regresión se corre una regresión por mínimos cuadrados no lineales con las variables educativas, de corrupción y de desarrollo humano. Para la variable primaria, los coeficientes tienen los signos esperados, aunque son poco significativos. De esta forma, parece que el producto regional por estado se muestra poco sensible al capital humano regional, al considerar la corrupción.

La tendencia en las diferencias en corrupción muestra una convergencia significativa, al considerar ciertos niveles de educación y el índice de desarrollo humano. Sin embargo, el resultado importante es el de largo plazo, donde se puede afirmar que en los años 1970 a 2001, se observa una tendencia de convergencia en el nivel de ingreso per cápita promedio, condicional a la variable de corrupción, la 
cual aun se ha reflejado en la tasa de convergencia del PIB per cápita. Asimismo, es interesante notar que al considerar las variables de frontera y de corrupción al mismo tiempo, se observa el fenómeno de la divergencia en los estados de la república mexicana. El coeficiente de la regresión $D$ del ingreso per cápita a nivel es de 0.0184, lo que muestra divergencia regional en el periodo 1970-2001, al considerar la corrupción y una variable de estado fronterizo. La variable de corrupción es negativa y significativa en esta regresión, lo que nos da una idea de un efecto negativo con el crecimiento del ingreso per cápita en los estados de la república mexicana para el periodo 1970-2001, pues por cada 100 pesos que se incrementa el ingreso per cápita, por lo menos uno de ellos es dedicado para actividades de corrupción dentro dela tramitología burocrática de M éxico. Esto es congruente con estudios gubernamentales que mencionan que la corrupción dentro de la normatividad en las compras que realiza el gobierno y los servicios que éste contrata, puede llegar a ser hasta de 57000 millones de pesos anuales, equival ente a 5500 millones de dólares, pero que aun así resulta una estimación moderada de lo que le cuesta al país la corrupción dentro de las instituciones (cuadro 3).

En la regresión $D$ la variable de estado perteneciente a la frontera norte es negativa y significativa, por lo que podemos observar que los estados de la frontera crecen de manera desigual que otros estados de México. Al incluir esta variable en el estudio empírico se obtiene un resultado de divergencia en el modelo de crecimiento para México. Esto nos puede dar una idea de que los estados de la frontera norte no convergen en el largo plazo con los estados más pobres de México.

El estadístico $\mathrm{F}$ de las regresiones son significativas. Con un vaIor $p$ bajo de 0.05 , y para una $F(2,28)$, se muestra un valor crítico igual a 3.34, lo cual indica que la hipótesis nula de que todos los coeficientes estimados son iguales a cero se puede rechazar, por lo que el resultado es a favor de la hipótesis alternativa de que al menos un coeficiente del modelo no es igual a cero y que el modelo es significativo. 
CUADRO 3. Estadísticasestatalesdel estudio (1970-2001).

\begin{tabular}{|l|c|c|c|c|c|c|}
\hline & Corrupción & I D H & Índice educativo & Iogy1970 & Iogy2001 & Cambi ol ogy \\
\hline A guascalientes & 4.53 & 0.563 & 0.846 & 0.89817648 & 1.258857 & 0.36068051 \\
Baja California & 5.74 & 0.5675 & 0.8602 & 1.16286299 & 1.27753314 & 0.11467014 \\
Baja California Sur & 3.88 & 0.5691 & 0.8511 & 1.14457421 & 1.29807319 & 0.15349899 \\
Campeche & 7.34 & 0.5644 & 0.7599 & 0.92427929 & 1.36485081 & 0.44057152 \\
Coahuila & 4.99 & 0.5737 & 0.8614 & 1.07990447 & 1.30949919 & 0.22959472 \\
Colima & 2.96 & 0.5486 & 0.8173 & 0.93500315 & 1.16379316 & 0.22879001 \\
Chiapas & 6.77 & 0.4546 & 0.6308 & 0.6946052 & 0.83902138 & 0.14441618 \\
Chihuahua & 5.51 & 0.5518 & 0.8362 & 1.00603795 & 1.34428008 & 0.33824213 \\
Distrito Federal & 22.62 & 0.6208 & 0.9067 & 1.28555731 & 1.57670428 & 0.29114697 \\
Durango & 8.85 & 0.55 & 0.8185 & 0.85672889 & 1.0978395 & 0.24111061 \\
Guanajuato & 5.95 & 0.5113 & 0.7372 & 0.85369821 & 1.02218195 & 0.16848374 \\
Guerrero & 13.39 & 0.4748 & 0.666 & 0.71432976 & 0.87851167 & 0.16418191 \\
Hidalgo & 6.67 & 0.4874 & 0.7209 & 0.73158877 & 0.98544347 & 0.25385471 \\
Jalisco & 11.55 & 0.5516 & 0.8129 & 1.01745073 & 1.15853391 & 0.14108318 \\
México & 16.95 & 0.5504 & 0.8328 & 1.03422726 & 1.0738378 & 0.03961054 \\
Michoacán & 10.31 & 0.5039 & 0.7369 & 0.72098574 & 0.93792571 & 0.21693996 \\
Morelos & 7.65 & 0.5485 & 0.8075 & 0.92634245 & 1.09309132 & 0.16674887 \\
Nayarit & 6.43 & 0.5463 & 0.7939 & 0.87966921 & 0.93183727 & 0.05216806 \\
Nuevo León & 7.05 & 0.6091 & 0.8915 & 1.22297645 & 1.41157386 & 0.18859741 \\
\hline
\end{tabular}




\section{CUADRO3.Continúa}

\begin{tabular}{|l|c|c|c|c|c|c|}
\hline & Corrupción & IDH & Índice educativo & logy1970 & Iogy2001 & Cambiol ogy \\
\hline Oaxaca & 7.36 & 0.4418 & 0.6609 & 0.54777471 & 0.80133312 & 0.25355841 \\
Puebla & 12.1 & 0.4866 & 0.722 & 0.79448805 & 0.99698153 & 0.20249348 \\
Querétaro & 8.05 & 0.5305 & 0.7893 & 0.89652622 & 1.25843024 & 0.36190402 \\
Quintana Roo & 6.12 & 0.5371 & 0.7173 & 1.00130093 & 1.38130949 & 0.38000855 \\
San Luis Potosí & 5.73 & 0.5261 & 0.7556 & 0.76715587 & 1.02933337 & 0.2621775 \\
Sinaloa & 7.81 & 0.5531 & 0.8137 & 0.97220284 & 1.06555427 & 0.09335143 \\
Sonora & 5.53 & 0.5648 & 0.8557 & 1.14270225 & 1.22375112 & 0.08104887 \\
Tabasco & 8.54 & 0.5168 & 0.7785 & 0.86153441 & 0.95463343 & 0.09309902 \\
Tamaulipas & 6.29 & 0.5615 & 0.8425 & 1.02201574 & 1.21070951 & 0.18869377 \\
Tlaxcala & 6.59 & 0.5235 & 0.8099 & 0.65896484 & 0.91745436 & 0.25848951 \\
Veracruz & 7.93 & 0.5109 & 0.7392 & 0.91115761 & 0.94671057 & 0.03555296 \\
Yucatán & 6.77 & 0.5209 & 0.743 & 0.85672889 & 1.08268368 & 0.22595479 \\
Zacatecas & 6.2 & 0.5299 & 0.7722 & 0.7126497 & 0.9009654 & 0.1883157 \\
\hline
\end{tabular}


En M éxico se ha comprobado empíricamente la teoría del modelo de crecimiento con evidencia de convergencia y divergencia entre los estados de nuestro país, considerando la corrupción, que tiene un efecto negativo en la tasa de crecimiento del producto per cápita, por lo que debe esperarse una tendencia a cerrar la brecha entre los estados ricos como el Distrito Federal, Baja California, Jalisco y Nuevo León, y los estados pobres, como Chiapas, Oaxaca y Guerrero. La convergencia en el ingreso estatal considerando variables de corrupción, educativas y de desarrollo humano, sin embargo, podrá tomar entre 45 a 100 años, o lo que es lo mismo, todo el siglo XXI.

\section{CONCLUSIONES}

La introducción de nuevas teorías del crecimiento y desarrollo regional ha consolidado nuestra capacidad de análisis de la economía regional. El presente estudio describe las principales escuelas de pensamiento enfocadas al crecimiento económico regional, con énfasis en el análisis de las instituciones. Sin embargo, algunas escuelas de pensamiento importantes no recibieron la representación justa, tal como teorías dinámicas no monotónicas del crecimiento (en donde las regiones con niveles de ingresos medios pueden crecer más rápidamente que regiones ricas o pobres), pero se muestra la importancia de la región geográfica en los éxitos del crecimiento, como lo es la variable de estado de frontera norte en México.

Los últimos veinte años traen una profunda reestructuración de la economía regional y el papel de los individuos y de los gobiernos regionales. Romer (1993) y Olson (1996) destacan el papel de las instituciones en facilitar el uso de ideas mediante externalidades positivas en la sociedad, y discuten que la economía se debe enfocar en el papel de las instituciones, las ideas y las innovaciones.

Para el periodo analizado en este estudio, que abarca de $1970 \mathrm{a}$ 2001, se muestra cierta convergencia debido a que las economías de los estados más atrasados (en el periodo inicial de 1970) crecen, en promedio, a un ritmo mayor que los estados más avanzados. 
La evidencia empírica reciente para México, sugiere que un factor dominante que distingue el elevado crecimiento de algunas regiones y el bajo crecimiento de otras, es la calidad del gobierno; es decir, la calidad de sus instituciones económicas, sociales y políticas.

El estudio demuestra que el gobierno y la corrupción en México están muy ligados, y esto diferencia a las regiones del país en términos decrecimiento. La corrupción seapodera del buen gobierno, y el mal gobierno produce mayor corrupción, y esto puede tener efectos negativos en el crecimiento económico de México y la convergencia entre las regiones. Final mente, se observa el efecto de la divergencia económica al considerar la variable de estado fronterizo.

\section{BIBLIOGRAFÍA}

Abramovitz, M. 1986. "Catching-up, forging ahead, and falling behind". Journal of Economic History, vol. 46, núm. 2, pp. 385-406. Bardhan, P. 1997. "Corruption and development: A review of issues". Journal of Economic Literature, vol. XXXV, Septiembre, pp. 13201346.

Barro, Robert J. 1989. "Economic growth in a cross section of countries". N ational Bureau of Economic Research Working Paper \#3120.

1990. "Government spending in a simple model of endogenous growth". Journal of Political Economy 98, pp. S103-S125.

1991. "Economic growth in a cross section of countries". Q uarterly Journal of Economics, vol. CVI, Mayo, pp. 407-443. 1996. "Democracy and growth". Journal of Economic Growth, 1, pp. 1-27.

1997. "Determinants of economic growth: A cross-country empirical study". H arvard Institute for International D evelopment Discussion Paper \#579.

Barro, Robert J. y J. Lee. 1993. "International comparisons of educational attainment". Journal of M onetary Economics 32, : pp. 363-394. 
Barro, Robert J. y Jong-Wha Lee. 1994. "Sources of economic growth (with comments from Nancy Stokey)". Carnegie-Rochester Conference Series on Public Policy 40, pp. 1-57.

Barro, Robert J. y Xavier Sala-i-Martin. 1990. “Economic growth and convergence across the United States". W orking Paper 3419, NBER, Agosto.

1991. "Convergence across states and regions". Brookings P apers on Economic Activity, 1, pp. 107-182.

1992. "Convergence". Journal of Political Economy 100(2), pp. 223-251.

1995. Economic growth. McGraw Hill. New York.

Caselli F, G. Esquivel y F. Lefort (1996). "Reopening the convergence debate: A new look at cross-country growth empirics". Journal of Economic Growth, 1, pp. 363-389.

Cellini, R. 1997. "Growth empirics: Evidence from a panel of annual data". A pplied Economic Letters, 4, pp. 347-351.

Data Bases, Foreign Broadcast Information Service (FBIS). United States Government. IPU Parline Database. http:/ / www.ipu.org/ parline . Data Base. http:/ / www.ifes.org/ eleccal .Political

Díaz Bautista, Alejandro. 1999. "Convergence, human capital and growth". Working, artículo DTE 7/ 99. Departamento de Estudios Económicos. El Colegio de la Frontera Norte. México. 2000. "Convergence and economic growth in M exico". Frontera N orte, vol. 13, pp. 85-110, julio-diciembre.

2001. "An introduction to institutional change, corruption and economic growth". Cuaderno de Trabajo, DTE 35/ 01. Departamento de Estudios Económicos. El Colegio de la Frontera Norte. México.

2002. "The role of telecommunications infrastructure and

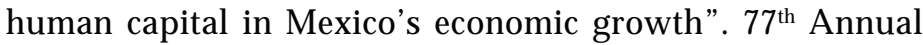
Conference of the Western Economic Association. Seattle, Washington, Julio 1.

De La Fuente, A. 1995. "Inversión, catch-up tecnológico y convergencia real". Papeles de Economía Española, núm. 63, pp. 18-34. 
De Long, J. Bradford. 1988. "Productivity growth, convergence, and welfare: Comment". A merican E conomic Review, vol. 78, núm. 5, diciembre, pp. 1138-1154.

De Long, J. Bradford y Lawrence H. Summers. 1993. “How strongly do developing economies benefit from equipment investment?". Journal of M onetary Economics 32(3), pp. 395-415. 1991. "Equipment investment and economic growth". Q uarterly Journal of Economics, vol. 106, núm. 425, pp. 445-502.

Dowrick, Steve y Duc-Tho Nyugen. 1989. “OECD Comparative Economic Growth 1950-85: Catch-up and convergence". A merican Economic Review, vol. 79, núm. 5, pp. 1010-1030.

Easterly, William. 1993. "How much do distortions affect growth?".

Journal of M onetary Economics, 32(2), pp.187-212.

Esquivel, Gerardo. 1999. "Convergencia regional en México". El trimestre económico, vol. LXVI, octubre-diciembre. México.

Hansen, Alvin H. 1950. "A contribution to the theory of the trade cycle". Oxford University Press, Oxford.

Harrison, Ann. 1995. "Openness and growth: A time-series, crosscountry analysis for developing countries". NBER Working Paper \#5221.

Hirschman, A. D. 1958. The Strategy of Economic D evelopment. Yale University Press. New Haven, Connecticut.

Human Development Index. 2001. U nited N ations H uman D evelopment Report 1996-2001. Oxford University Press. Oxford.

INEGI. VIII, IX, X, XI Censo general de población y vivienda. Instituto Nacional de Estadística, Geografía elnformática. México. 1960, 1970, 1980, 1990, 2000 y datos de la página electrónica.

Krugman, Paul. 1991. Geography and Trade. MIT Press.Cambridge, Massachusetts.

1995. D evelopment, Geography and Economic Theory. MIT Press. Massachusetts.

Krugman, Paul, Fujita y Venables. 1999. “Thespatial economy: Cities, regions, and international trade". MIT Press.

Katz, Issac, M. 1999. “La Constitución y el desarrollo económico de México", 1a. edición, Cal y Arena. 
Kauffman, Daniel. 1997. “Corruption: The facts”. Foreign Policy, 107, pp. 114-131.

Keynes, John Maynard. 1936. "The general theory of employment, interest, and money". Harcourt Brace. Londres.

Keefer, Philip y Stephen Knack. 1997. “Why don't poor countries catch up? A cross-national test of an institutional explanation". Economic Inquiry 35, pp. 590-602.

Knack, Stephen y Philip Keefer. 1995. "Institutions and economic performance: Cross-country tests using al ternative measures". Economics and Politics, 7 (3), pp. 207-227.

Lucas, Robert E. 1988. "On themechanics of economic development". Journal of M onetary Economics 22, pp. 3-42.

Mankiw N. Gregory. 1995. "The growth of nations". Brookings Papers on Economic Activity, 1:1995, pp. 275-326.

Mankiw, N. Gregory y David Romer. 1991. “New Keynesian economics". MIT Press. Cambridge.

Mankiw, N. Gregory, David Romer and David N. Weil. 1992. "A contribution to the empirics of economic growth". Q uarterly Journal of Economics 107(2), pp. 407-437.

Mauro, Paolo. 1995. "Corruption and growth". Q uarterly Journal of Economics 110(3); pp. 681-713.

Mauro, Paolo. 1997. “Why worry about corruption?". Economic Issues 6. IMF Washington.

1998. "The effects of corruption on growth, investment, and government expenditure". IM F W orking Paper 96/ 98. IMF's Policy Development and Review Department.

Messmacher, Miguel. 2000. “Desigualdad regional en México. El efecto del TLCAN y otras reformas estructurales". Documento de Investigación núm. 2000-4. Dirección General de Investigación Económica. Banco de México. Diciembre, pp. 1-33.

Murphy, Kevin M., Andrei Shleifer y Robert W. Vishny. 1991. "The allocation of talent: Implication for growth". Quarterly Journal of Economics 106(2), pp. 503-530.

Myrdal, Gunnar. 1968. A sian D rama: An Inquiry into the Poverty of $\mathrm{N}$ ations. Twentieth Century Fund. Nueva York. 
North, Douglass C. 1990, 1991. Institutions, Institutional Change, and Economic Performance. CambridgeUniversity Press. Cambridge, Maassachusetts.

1993. Institutions and Productivity in History. Washington University. Washington.

1994. Institutional Change: A Framew ork of A nalysis. Washington University. Washington.

1997. “Prologue”. In John N. Drobak and John V. C. Nye (eds.). The Frontiers of the $\mathrm{N}$ ew Institutional Economics. A cademic Press. San Diego.

Olson M. 1996. "Big bills left on sidewalk: Why some nations are rich, and others poor". Journal of Economic Perspectives, vol. 10, núm. 2, pp. 2-24.

Organization for Economic Cooperation and Development. 1995. A ssessing Structural Change: Lessons for the Future. OECD. París. Porter, Michael. 1990. "The Competitive Advantage of Nations". The Free Press.

Quah Danny T. 1993. “Empirical cross-section dynamics in economic growth". European Economic Review, 37, pp. 426-434.

1996. "Convergence empirics across economies with (some) capital mobility". Journal of Economic Growth 1, pp. 95-124.

Rodrik, Dani. 2000. "Institutions for high-quality growth: What they are and how to acquire them", N BER Working Paper núm. W7540.

Romer Paul M. 1990. "Human capital and growth: Theory and evidence". Carnegie-Rochester Conference Series on Public Policy, 32, pp. 251-286.

1990. “Endogenous Technological Change”. Journal of Political E conomy 98, S71-S102.

1993. Two Strategies to Economic D evelopment: U sing Ideas and Producing I deas. Proceedings of World Bank A nnual Conference on Development.

1993. "Idea gaps and object gaps in economic development". Journal of M onetary Economics 32(3), pp. 543-573.

1994. "The origins of endogenous growth". Journal of Economic Perspectives, 8(1), pp. 3-22. Invierno. 
Sachs, Jeffrey D. 1997. "Nature, N urture, and Growth". TheE conomist, 14 de junio, pp. 19-21.

Sachs, Jeffrey D. y A ndrew M. Warner. 1997. "Sources of slow growth in african economies". Journal of A frican Economies, 6 (3), pp. 335-376.

1995. "Economic reform and the process of global integration (with comments and discussion)". Brooks Papers on Economic Activity 1 1-118.

Sala-i-Martin, Xavier. 1994. "Apuntes de crecimiento económico". Antoni Bosch Editor, Barcelona.

1996. "The classical approach to convergence analysis". Economic Journal, 106, pp. 1019-1036.

1997. "I just ran two million regressions". AEA Papers and Proceedings 87, pp. 178-183.

Secretaría de Educación Pública. 2002. Estadísticas Educativas and Secretaría de Educación Pública (SEP). http:/ / www.sep. gob.mx.

Schleifer, A. y R. Vishny. 1993. "Corruption". The Q uarterly Journal of Economics 108(3), pp. 599-617.

Smith, Adam. 1776. A $n$ inquiry into the nature and origins of the wealth of nations. Modern Library, Nueva York. 1937.

Solow, R. M. 1956. "A contribution to thetheory of economic growth". Q uarterly Journal of Economics, febrero, pp. 65-94.

1957. "Technical change and the aggregate production function". Review of Economics and Statistics, 39, pp. 312-320.

Summers, R. y Heston A. 1991. "The Penn World Table (Mark 5): An expanded set of international comparisons, 1950-1988". Quarterly Journal of Economics, mayo, pp. 328-368.

1995. "Penn World Tables 5.6". University of Toronto. http/ / cansim.epas.utoronto.ca:5680/ pwt/ pwt/ .html

Tanzi, V. 1998. "Corruption around the world: Causes, consequences, scope, and cures". IM F Staff Papers, 45 (4), diciembre, pp. 559-594.

Transparencia Internacional. 2001. Encuesta Nacional de Corrupción y Buen Gobierno. 
Transparency International. 2000. Press R el ease: Transparency International Releases the Y ear 2000 Corruption Perceptions Index. Septiembre 13, Berlín.

Transparency International. 2001. The Integrity Pact (TI-IP). The Concept, the M odel and the Present A pplications. A Status Report. UNESCO Statistical Yearbook. Varios años. Organización de las Naciones Unidas, París.

U nited N ations International Financial Yearbook. 1979, 1994. Organización de las Naciones Unidas. Nueva York.

U nited $\mathrm{N}$ ations Development Programme. Human Development Report. Varios años. Oxford University Press. Nueva York.

World Bank. 1997. W orld Development Report 1997: The state in a changing world. Oxford University Press for the World Bank. Washington, D.C.

1997. Helping countries combat corruption. The role of the world bank. PREM. Washington, D.C.

1999. "Anticorruption strategy for Revenue Administration". PREM Note No. 33, Octubre. Washington, D.C. 2000. "Anticorruption in Transition". A Contribution to the Policy D ebate. Washington, D.C. 